19

\section{What Are the Projective Fields of Cortical Neurons?}

Terrence J. Sejnowski

\section{What is the Receptive Field of a Neuron?}

The concept of receptive field is central to understanding the response properties of sensory neurons. It grew out of early studies on sensory receptors by Sherrington (1906), in which he defined an "adequate" stimulus as one that causes the receptor to respond in a specific manner, such as light for photoreceptors, and the receptive field of the receptor as the spatial region of the sensory surface over which the adequate stimulus generates a response. The receptive field of a central neuron is an extension of this concept and is defined as the region of the sensory surface which when stimulated by an adequate stimulus produces an excitatory response. The stimulus that elicits the strongest response is used to define the trigger feature of the cell, which has led to the view of cortical cells as feature detectors.

The receptive field continues to be highly relevant for current experimental studies of the cortex, but it has evolved over the years as we have learned more about cortical neurons. First, the response to the presentation of a sensory stimulus is not purely passive as previously assumed: the response can be modulated by attention and reward expectation (Rries et al. 2001; Richmond, liu, and Shidara 2003). Moreover, cortical neurons have intrinsic properties that allow neurons to generate activity without an external sensory stimulus (Llinas 1988). Second, there are regions outside the classically defined receptive field that can modulate the response of the cell (Allman, Miezin, and McGuinness 1985; Movellan et al. 2002). These surround influences take into account the context of the stimulus, such as the modulation of perceived color by the color of the surround, which is important for color constancy under varying illumination (Wachtler, Albright, and Sejnowski 2003).

The receptive field, as modified by recent discoveries, is still used today to experimentally characterize cortical neurons. Knowing the receptive field properties of a neuron helps to understand its possible function in the brain. For example, a neuron that has a visual receptive field and prefers vertical edges presumably has a function that is visual and is related in some way to analyzing vertical edges. However. knowing only the receptive field of a neuron is not sufficient to conclude what its function is in the brain.

\section{What is the Projective Field of a Neuron?}

The focus of this chapter is on properties of a neuron that are complementary to those that characterize the receptive field. In addition to knowing what stimulus excites a neuron, it is equally important to know what the impact that neuron has on downstream targets. This is called the "projective field" of a neuron (Lehky and Sejnowski 1988), which will be defined more precisely later, but first we need to motivate the need for the new concept. 
Over a hundred years ago, neurologists had determined the projection pathways between different cortical areas and had begun to speculate about their functions (Wemicke 1900). Pathways were traced from the periphery to the cortex and between cortical areas. This was a form of "connectionism" in which the information stored in different parts of the cortex could be linked by direct pathways joining them. More recently, single-unit recording techniques have been used to establish the properties of the projection cells and to show how their properties are transformed along the pathways (Hubel and Wiesel 1962).

In 1.988 Sidney Lehky and I developed a neural network model based on the early stages of processing in the visual system whose function was to compute the shape, or more precisely the curvature, of a curved surface from its shaded image (Lehky and Sejnowski 1988). This is called the "shape-fromshading" problem in computer vision. The details about how the network was constructed are not important here; what is interesting is that many of the model neurons in the network had receptive field properties that were similar to the simple cells in the primary visual cortex of monkeys. Simple cells have excitatory and inhibitory subregions that form an oriented spatial filter.

In addition to testing each of the neurons in the model with the same stimuli used by visual physiologists to characterize the receptive fields of real neurons, we were able examine how each neuron affected the output layer, which represented the curvature of the shaded object in the input image. Some of the model neurons that resembled simple cells were indeed behaving like a filter and provided information about the orientation of the curved surface; however, others had an entirely different function and instead served as detectors for the direction of the illumination and sign of the curvature. They tended to have a bimodal distribution of firing rates, at either a low rate or a high rate of firing.

The shape-from-shading network model demonstrated first that the visual system was capable of performing this computation and second that it was not possible to deduce the function of a neuron solely from its receptive field properties. In addition, the connections of the neuron to the output were also needed, which we called the projective field of the neuron. In retrospect, it is obvious that a neuron without any output cannot have a computational function and that the same neuron can have more than one function depending on where it projects and what that information is used for. This raises the interesting question of how to experimentally determine the projective fields of neurons in different parts of the brain and whether this will provide further insight into the functions of single neurons. This chapter is an exploration of this question.

\section{How Can the Projective Field of a Neuron Be Measured?}

The receptive field of a neuron depends on its synaptic inputs; in contrast, the projective field of a neuron depends on its axonal arborization. Thus, one source of evidence about the projective field of a neuron is its axonal targets. For example, a clue to the function of a cortical neuron may be found if it projects to a motor structure, such as the ventral horn of the spinal cord. However, in addition to knowing where a neuron projects, it is equally important to know the impact that the fring of the neuron has on the downstream target neurons. This aspect of neural function can be approached with stimulation techniques.

In an ideal experiment, a single neuron would be stimulated to assess its impact by comparing a behavioral measure with and without stimulation. This is a technically difficult experiment, but several groups now have used patch recording of cortical neurons in vivo, so it is at least feasible. When a single neuron in the deep layers of rat barrel cortex was stimulated with a train of stimulus, it produced a sequence of small coordinated movements in multiple whiskers that outlasted the stimulus (Brecht et al. 2004). The effectiveness of the stimulus was enhanced during waking states. This suggests that minimal size of a group of neurons that can support a complex behavioral state could be on the low side, perhaps as low as a few dozen.

With extracellular stimulation more than one neuron is activated, but it is not known how many or how strongly they are activated. For example, microstimulation of the motor cortex, designed to produce a minimal muscular contracture, was used to map out the different body regions on the surface of the cortex (Asanuma, Arnold, and Zarzecki 1976). It is interesting that extracellular stimulation of neurons in the visual system, such as the frontal eye fields in prefrontal cortex, and even the primary visual cortex, can stimulate eye movernents to the corresponding region of the visual field that was stimulated (Pouget, Fisher, and Sejnowski 1993).

More recently, extracellular stimulation of parts of the visual cortex of awake monkeys has been used to alter their perceptual judgment. In visual area $\mathrm{MT}$, with neurons that respond selectively to the direction of motion, electrical stimulation can bias the monkey to respond in the preferred direction of the neurons in the column that is being stimulated (Salzman et al. 1992). It is not known whether the perception or the action plan of the monkey is altered by this stimulation, since we do not have access to a monkey's visual awareness (Crick and Koch, chapter 23, this volume).

Perhaps the most dramatic example of the impact of stimulating cortical neurons occurs in humans during brain surgery to prevent epilepsy (Penfield and Roberts 1959). Electrical stimulation was used to map out the cortical regions that need to be spared during surgical resection, such as the language areas (figure 19-1). Stimulation of cortical areas in awake patients sometimes evoked sensory percepts and vivid recollections of past events:

When electrical stimulation recalls the past, the patient has what some of them have called a "flash-back." He seems to re-live some previous period of 
time and is aware of those things of which he was conscious in that previous period. It is as though the stream of consciousness were flowing again as it did once in the past. (p. 45)

These early studies used a crude surface electrode and high currents to stimulate the cortex. This almost certainly resulted in current spread, so that the actual neurons that were stimulated could not be determined. At high currents, cortical neurons directly under the electrode are blocked from

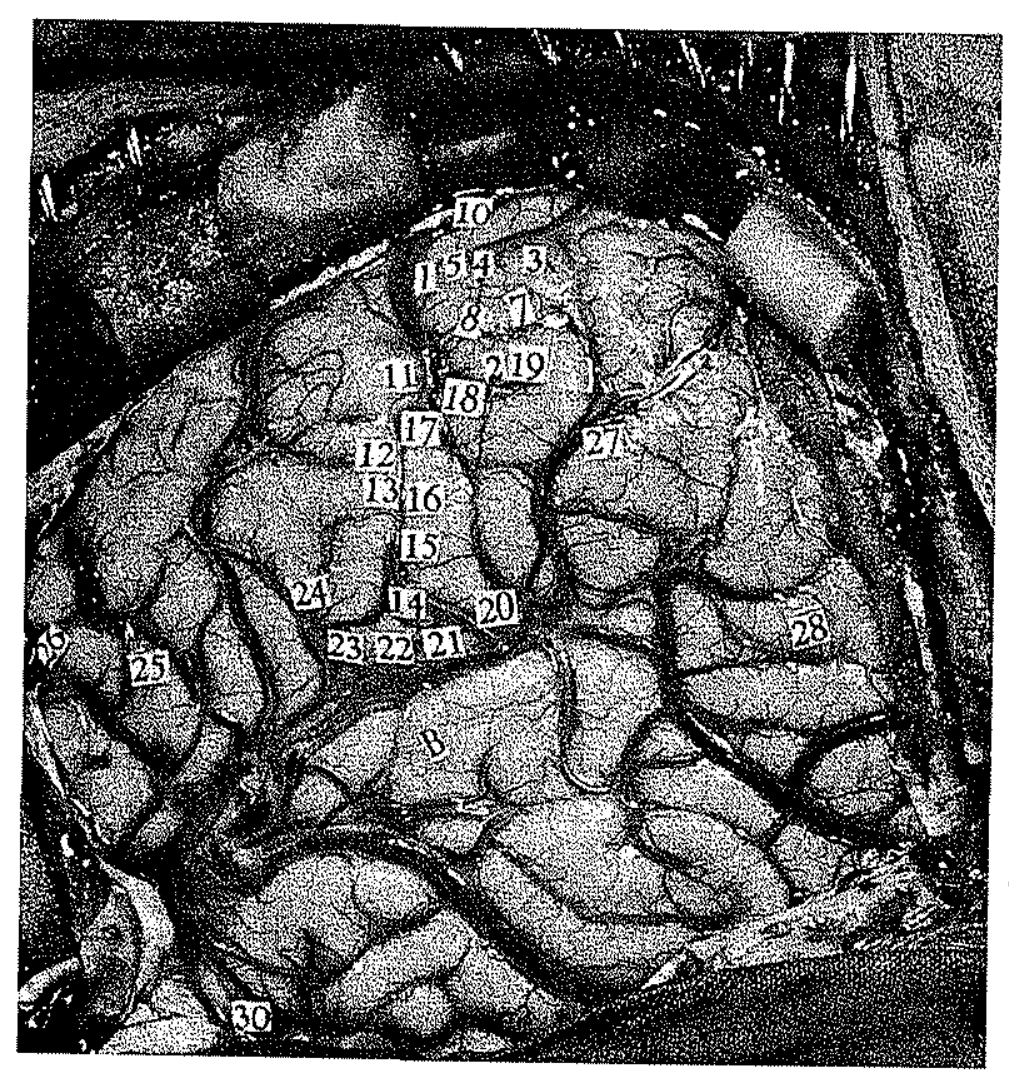

Figure 19-1. During an operation to cut out the epileptic locus, different parts of the cortex are stimulated to identify speech areas. In this patient, the brain is exposed over the left temporal lobe and the numbered tickets, dropped onto the surface of the cortex, indicate points of positive responses to electrical stimulation. Some of the responses to the stimulation were: 16, tingling in the tip of the tongue 21 , opening of the jaw; 27 , the patient said, "Oh, I know what it is. That is what you put in your shoes." After withdrawal of the electrode he said, "foot"; 30 , patient tried to talk but could not. Reproduced with permission from Penfield (1959).

398 Organization of Cognitive Systems firing, and the activated neurons were probably located in a large penumbra surrounding the site of stimulation. At best, these experiments provide only hints about the consequences of stimulating smaller populations of neurons.

The Projective Fields of Networks of Neurons in the Motor Cortex

Ideally, the stimulus strength and duration should not be too low or too high in order to recruit assemblies of neurons typical of those that are active during normal brain states. In a study of neurons in the motor cortex, Graziano, Taylor, and Moore (2002) explored the impact of stimulating neurons in the motor cortex of monkeys with trains of stimuli similar in frequency and duration to the activity recorded in the motor cortex during a limb movement. This level of stimulation produced coordinated contractions in a set of muscles. When the arm region was stimulated, complex arm movements were observed that resulted in a change in the posture of the arm relative to the body of the monkey. When the same location was stimulated, regardless of initial arm position the arm of the monkey ended in the same posture, as shown in figure 19-2. The map of the motor cortex using this pattern of stimulation was a map of body postures rather than a map of individual muscles.

Because many neurons in the same column were stimulated at the same time, it is likely that many other neurons were recruited from brain areas to which these neurons projected. These experiments have been criticized because of the uncertainty about which neurons were being stimulated, but from the perspective of the projective field of a neuron, this is precisely what we want to discover. Just as it is important to discover the "adequate" sensory stimulus to map out the receptive field of a neuron, it is also important to discover the "adequate" stimulation pattern to map out the projective field of a neuron. A train of stimuli corresponding to its natural pattern of activity is more likely to provide adequate stimulation than minimal stimulus. After all, the stimulus used to map the receptive field is not a minimal stimulation. but one that produces the maximal response of the neuron.

Another issue that these experiments raise is the question of whether we should be thinking in terms of individual neurons or populations of neurons in defining the projective field. It is rare that a single neuron will fire in a column of neurons in response to sensory stimulus or during a motor action, so it might be best to focus on the projective field of a network of interacting neurons that typically act together. One of the advantages of the columnar architecture that has been observed throughout the cortex is that neurons with similar properties are often located nearby. 
IPSILATERAL.
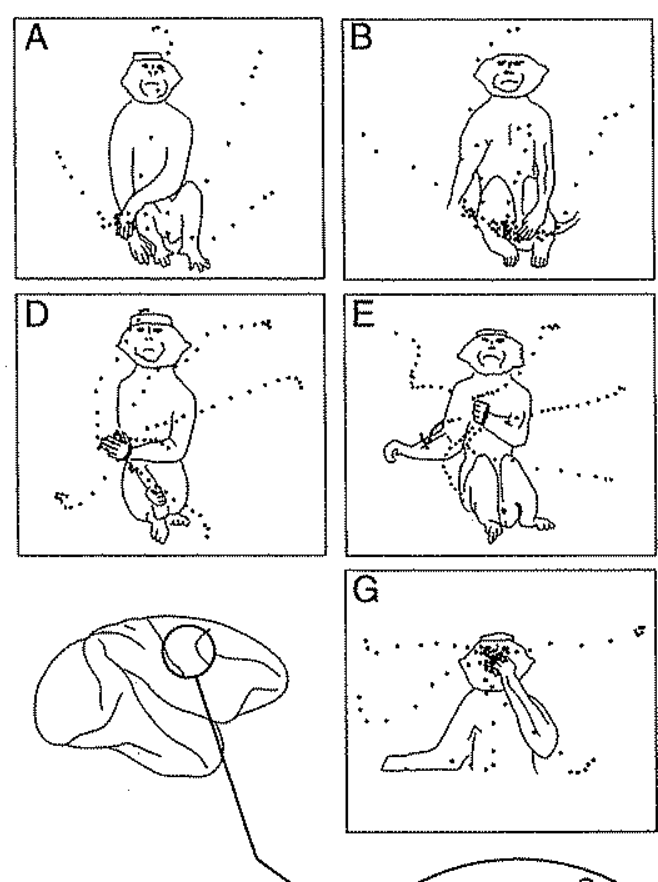

\section{H}
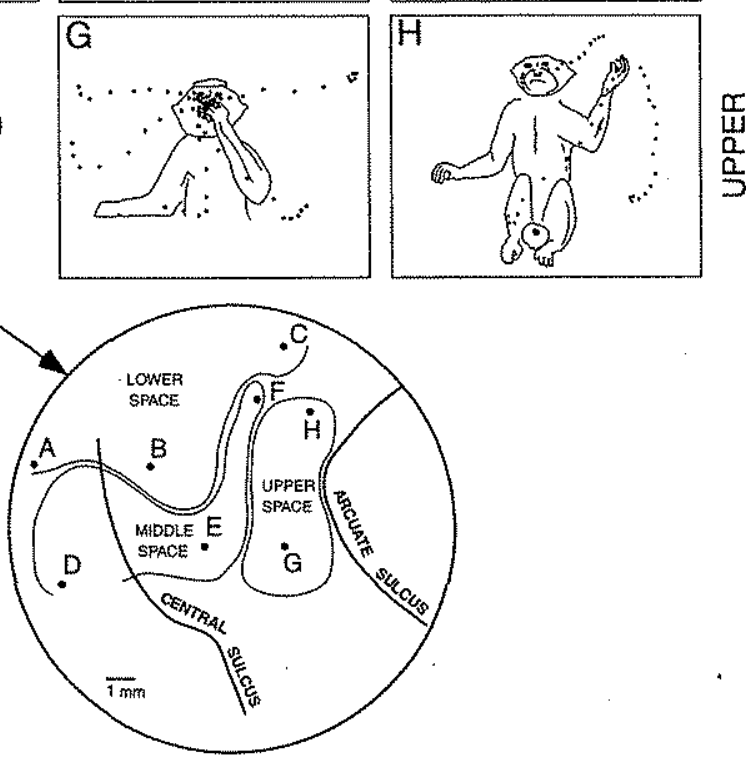

Figure 19-2. Characteristic postures produced by stimulating different regions of the precentral cortex of a monkey. The magnified view at the bottom shows the locations of the stimulation sites. The area to the left of the lip of the central sulcus represents the anterior bank of the sulcus. Stimulation on the right side of the brain caused movements mainly of the left side of the body. For all sites,

stimulation trains were presented for $500 \mathrm{~ms}$ at $200 \mathrm{~Hz}$. Adapted from Graziano, Taylor, and Moore (2003).

\section{The Penfield Project}

In view of this recent work in the motor cortex, the pioneering experiments by Wilder Penfield take on a renewed interest. Could it be that Penfield had been able to stimulate the projective fields of cortical networks and that his anecdotal reports of complex streams of thought were "cognitive postures" similar to the muscular posture elicited by a train of stimuli in the monkey motor cortex? It is not possible to come to any conclusion based on the few extant reports, but similar experiments could be undertaken today under more controlled conditions. It is now common practice to implant an array of electrodes on the surface similar to those used by Penfield and also depth electrodes in the hippocampus and amygdala. Recordings from single cortical neurons in humans are possible (Kreiman, Fried, and Koch 2002) and electrical stimulation also could be undertaken at the same time.

Similar explorations could be undertaken in monkeys, where much more is known about the properties of neurons in different cortical areas and the projections between them. The goal would be to identify which patterns of stimulation produce complex behaviors or can influence the performance of a monkey in a cognitive task. At the same time it should also be possible in monkeys to record simultaneously from several cortical areas to determine the extent to which the pattern of stimulation in one area spreads to other areas of cortex. The overall goal of characterizing the projective fields of neurons in this way could be called the "Penfield Project."

\section{Mirror Neurons}

Is it possible for the receptive field and projective field of a neuron to match each other? That is, what if the sensory stimulus that a neuron responds to is in some way related to the motor output that would be elicited by stimulating this neuron? Such neurons have been found in the prefrontal cortex and other brain areas. These neurons respond when a monkey observes complex actions, such as a precision grip of an object, and are also activated when the monkey makes the same action (Rizzolatti, Fogassi, and Gallese 2002). They are called mirror neurons because both their sensory responses and motor correlates match. Although stimulation experiments have not yet been performed for brain areas that contain mirror neurons, similar to those in motor cortex (Graziano, Taylor, and Moore 2002), it is likely that an adequate stimulus would evoke actions similar to those that make the neurons respond.

Mirror neurons appear to solve the inverse problem: given a desired goal defined in terms of a sensory state, such as reaching for an object, what motor commands should be given to achieve that state? Another example is the 
problem of pronouncing words in a foreign language. Phonemes that are not present in a native language are especially difficult to mimic. If there are mirror neurons in the language areas of the human brain that respond to specific phonemes and when activated produce the same phoneme, then it is possible that a mirror system for language can be used to bootstrap the basic elements of speech and communication (Rizzolatti and Arbib 1999). There are of course many other aspects of language that need to be explained, but the complexity of the articulation should not be underestimated, and a solution to the inverse problem for speech articulation goes a long way toward explaining some aspects of language acquisition.

The existence of mirror neurons raises the question of what aspects of human behavior may be learned by example. Mimicry is a form of supervised learning that does not require a detailed feedback for each muscle. With an internal mirror system, it is possible to learn by observing, which opens up a powerful way to gain skills through social interactions.

\section{Autonomy}

We are not simple stimulus-response machines, but many research protocols put subjects into response paradigms that do not allow for the flexibility that may be the most important feature of the cortex. To better understand the contribution of the cortex to behavior during unconstrained conditions, we need to go beyond the artificial dichotomy between the sensory and motor systems that is often imposed by experimental paradigms (Churchland, Ramachandran, and Sejnowski 1994).

It is now possible to record reliably from hundreds of neurons simultaneously from the brains of freely moving animals (Wilson and McNaughton 1993). When a rat is allowed to explore an environment, neurons in its hippocampus fire specifically to locations in the environment, called the "place fields" of the neurons. However, the responses of these neurons are not simple sensory responses, since the place fields are preserved in the dark. Furthermore, the activity patterns of a hippocampal neuron can be shifted by altering the environment or changing the task that the rat is expected to perform (Gothard et al. 1996). This suggests that neurons in the hippocampus are driven as much by internal states of the animal as by the outside world.

Ultimately we need to develop a more sophisticated way to understand the dynamics of the brain's internal states that are not dominated by either sensory inputs or motor actions, but by internal states that are autonomously generated. Autonomous brain activity, sometimes called spontaneous activity, has been known for over a century from electroencephalographic (BEG) recordings from the scalp. Autonomous brain activity is characterized by brain oscillations over a wide range of frequencies, which depend on the behavioral state of the animal (Destexhe and Sejnowski 2001).

Recordings of field potentials in monkeys suggest that some brain oscillations observed in the cortex may be related to attention and expectation (Fries et al. 2001). In humans, new methods for analyzing EEG recordings have revealed that the ongoing EEG may interact with sensory input, which can alter the phases of internal oscillations (Makeig et al. 2002). These recent discoveries about brain dynamics suggest that an important function of the thalamocortical system is to regulate the flow of information between brain areas, making it possible for the brain to rapidly respond to changing contingencies in the world and to anticipate these changes (Salinas and Sejnowski 2001). Rhythmic activity in the cortex continues during sleep states, whose function we are just beginning to appreciate (Sejnowski and Destexhe 2000).

\section{Conclusion}

As new methods are devised for recording from thousands of neurons simultaneously in widespread areas of primate brains (Carmena et al. 2003; Hofiman and McNaughton 2002) and as new methods are developed to analyze these signals (Zhang et al. 1998; Abbott and Sejnowski 1999), it should be possible to observe how the flow of information between brain areas is regulated by the behavioral state of the animal. In addition to characterizing the receptive field properties of neurons, it is also important to study their projective fields. The projective fields of interacting assemblies of neurons provide the basic "keyboard" for the brain's interaction with the world and with itself. By combining information about the receptive and projective fields of cortical neurons, an overall picture should emerge of how autonomous behaviors arise from dynamic brain states (Sejnowski 2003).

\section{References}

Abbott, L. F., and Sejnowski, T. J. (eds.). (1999) Neural Codes and Distributed Representations: Roundations of Neural Computation. Cambridge. Mass.: MTT Press.

Allman, J. (1999) Evolving Brains. New York: Scientific American Library,

Allman, J., Miezin, F, and McGuinness, E. (1985) Direction- and velocity-specific responses from beyond the classical receptive field in the middle temporal visual area. Perception 14: 105-126.

Asanuma, H., Arnold, A., and Zarzecki, P. (1976). Further study on the excitation of pyramidal tract cells by intracortical microstimulation. Exp. Brain Res. 26 , $443-461$.

Brecht, M. Schneider, M., Sakmann, B., and Margrie, T. W. (2004) Whisker movements evoked by stimulation of single pyramidal cells in rat motor cortex. Nature 427: 704-710. 
Carmena, J. M., Lebedev, M. A., Crist, R. B., O'Doherty, J. E., Santucci, D. M., Dimitrov, D., Patil, P. G., Henriquez, C. S., and Nicolelis, M. A. (2003) Learning to control a brain-machine interface for reaching and grasping by primates. PLOS Biol. 1(2): 193-208.

Churchland, P. S., and Sejnowski, T. J. (1992) The Computational Brain. Cambridge, Mass.: MTT Press.

Churchland, P. S., Ramachandran, V. S., and Sejnowski, T. J. (1994) A critique of pure vision. In: C. Koch and J. Davis (eds.), Large-Scale Neuronal Theories of the Brain, 23-60. Cambridge, Mass.: MIT Press.

Destexhe, A., and Sejnowski, T. J. (2001) Thalamocortical Assemblies: How Ion Channels, Single Neurons and Large-Scale Networks Organize Sleep Oscillations. Oxford: Oxford University Press.

Eries, P., Reynolds, J. H., Rorie, A. E., and Desimone, R. (2001) Modulation of oscillatory neuronal synchronization by selective visual attention. Science 291 : 1560-1563.

Gothard, K. M., Skaggs, W. E., Moore, K. M., and McNaughton, B. L. (1996) Binding of hippocampal CA1 neural activity to multiple reference frames in a landmark-based navigation task. J. Neurosci. 16(2): 823-835.

Graziano, M.S.A., Taylor, C.S.R., and Moore, T. (2002) Complex movements evoked by microstimulation of precentral cortex. Neuron 34: 841-851.

Hoffman, K. L., and McNaughton, B. L. (2002) Coordinated reactivation of distributed memory traces in primate neocortex. Science 297: 2070-2073.

Hubel, D. H., and Wiesel, T. N. (1962) Receptive fields, binocular interaction and functional architecture in the cat's visual cortex. ]. Physiol. 160: 106-154.

Kreiman, G., Fried, I., and Koch, C. (2002) Single neuron correlates of subjective vision in the human medial temporal lobe. Proc. Natl. Acad. Sci. USA 99: 8378-8383.

Laughlin, S. B., and Sejnowski, 'T. J. (2003) Communication in neural networks. Science 301: 1870-1874

Lehky, S., and Sejnowskl, T. J. (1988) Network model of shape-from-shading; Neural function arises from both receptive and projective fields. Nature 333: 452-454.

Llinas, R. R. (1988) The intrinsic electrophysiological properties of mammalian neuroins: Insights into central nervous system function. Science 242: 1654 1664.

Makeig, S., Westerfield, M. Jung, T..P. Enghoff, S., Townsend, J. Courchesne, B., and Sejnowski, T. J. (2002) Dynamic brain sources of visual evoked responses. Science 295: 690m694.

Movellan, J. R., Wachtler, T., Albright, T. D., and Sejnowski, T., (2002) Naive Bayesian coding of color in primary visual cortex. Adv. Neural Inf. Process. Syst. 15: 221-228.

Penfield, W., and Roberts, L. (1959) Speech and Brain-Mechanisms. Princeton: Princeton University Press.

Pouget, A., Fisher, S. A., and Sejnowski, T. J. (1993) Egocentric spatial representation in early vision. J. Cogn. Neurosci. 5: 150-161.

Quartz, S., and Sejnowski, T. J. (2002) Liars, Lovers and Heroes: What the New Brain Science Has Revealed About How We Become Who We Are. New York Harper-Collins.

Richmond, B. J., Liu, Z., and Shidara, M. (2003) Neuroscience: Predicting future rewards. Science 301: 179-80.
Rizzolatti, G., and Arbib, M. A. (1999) From grasping to speech: imitation might provide a missing link: reply. Trends Neurosci. 22(4): 152.

Rrotions of the ventral premotor cortex. Curr. Opin. Neurobiol. 12(2): 149-154.

Salinas, E., and Sejnowski, T. J. (2001) Correlated neuronal activity and the flow of neural information. Nat Rev Neurosci 2: 539-550.

Salzman, C. D. Murasugi, C. M., Britten, K. H., and Newsome, W. T. (1992) Microm stimulation in visual area MT: effects on direction discrimination performance. J. Neurosci. 12: 2331-2355.

Sejnowski, $\mathrm{T}$, . (1986) Open questions about computation in cerebral cortex. In: J. McClelland and D. Rumelhart (eds.), Explorations in the Microstructure of Cognition, Volume 2: Applications, 372-389. Cambridge: MT Press.

Seinowski, T. J. (2003) The computational self. Annals of the New York Academy of Sciences 1001: 262-271.

Sejnowski. T. J., and Destexhe, A. (2000) Why do we sleep? Brain Res. 886(1-2): 208-223.

Sherrington, C. (1906) The Integrative Action of the Nervous System. Cambridge: Cambridge University Press.

Wachtler, T., Sejnowski, T. J., and Albright, T. D. (2003) Representation of color stimuli in awake macaque primary visual cortex. Neuron 37:681-691.

Wernicke. C. (1900) Grundriss der Psychiatrie in klinischen. Leipzig, Ed Thieme.

Wilson, M. A., and McNaughton, B. L. (1993) Dynamics of the hippocampal ensemble code for space. Science, 261: 1055-1058.

Zhang, K., Ginzburg, I., McNaughton, G. L., and Sejnowski, T. I. (1998) Interpreting neuronal population activity by reconstruction: Unified framework with application to hippocampal place cells. J. Neurophysiol., 79: 1017-1044. 